\title{
Parés pour un avenir sur Internet
}

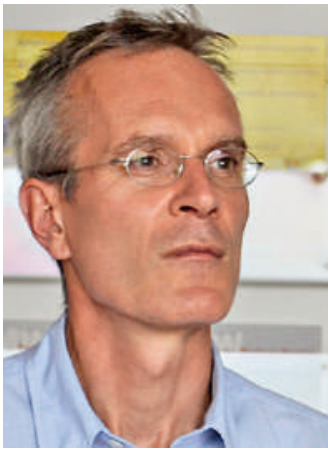

Bruno Kesseli
Si, en plus de la version papier du Bulletin des médecins suisses, vous consultez aussi de temps à autre, voire régulièrement, nos pages Web sur Internet (www.bullmed.ch), vous avez sans doute déjà constaté que, depuis quelques semaines, le BMS arbore une nouvelle présentation.

Dans un premier temps, les changements portaient surtout sur la mise en page et sur une structure de menu et une orientation du lecteur plus fonctionnelles (du moins nous l'espérons). Nous avons donc procédé à ce «relaunch» sans tambour ni trompette. Mais depuis plusieurs semaines que ces nouvelles pages Web sont opérationnelles et après nous être débarrassés de quelques maladies infantiles, nous souhaitons maintenant attirer votre attention sur certains aspects de ces changements, importants à nos yeux: configuration du site Internet. En cliquant sur le bouton «Get e-mail alerts» sur la page d'accueil du BMS, les visiteurs ont désormais la possibilité de s'abonner à une newsletter, avec un système d'alerte à la parution du dernier numéro du BMS, une synthèse des principaux articles et des liens directs vers les pages Web correspondantes.

Les grands titres du dernier numéro sont aussi affichés sur la page d'accueil, avec le plus souvent un bref résumé du contenu des articles. Ainsi, pour lire l'éditorial de la FMH, la chronique «Et encore» ou un article de fond du dernier BMS en ligne, il n'est plus nécessaire de passer par le sommaire. Le PDF souhaité peut être directement téléchargé. Le sommaire complet et le PDF de l'ensemble du numéro sont aussi accessibles d'un simple clic. Sous réserve d'avoir les compétences rédactionnelles nécessaires,

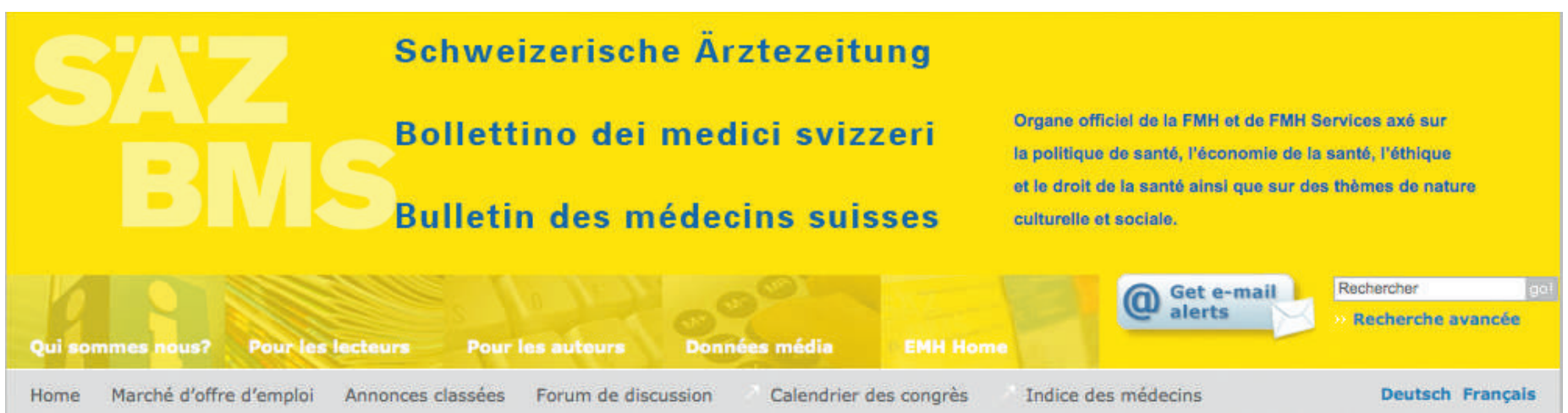

Le site Web du BMS est désormais basé sur un système de gestion de contenu performant et très flexible, TYPO3, qui peut être adapté au mieux aux besoins des différentes entreprises, mais aussi aux diverses attentes au sein d'une même entreprise, les Editions médicales suisses EMH et ses multiples revues par exemple. Ces adaptations sont possibles pour les exigences actuelles et futures, ce qui nous laisse une marge de manœuvre suffisante pour faire évoluer le site Web et introduire les nouvelles prestations souhaitables et nécessaires à long et à moyen terme.

Même si ce changement de système n'est pas visible (ou ne l'est que partiellement), il n'en reste pas moins la base sur laquelle le BMS va bâtir son avenir sur Internet. Conjointement avec un système de gestion des périodiques très performant lui aussi, l'efficacité de l'édition en ligne est considérablement améliorée grâce à l'automatisation de toute une série de processus auparavant exécutés manuellement.

Un certain nombre de nouveautés, suggérées ou souhaitées ces dernières années par les lecteurs, ont été mises en place en profitant de cette nouvelle l'outil évolutif «Newstool» du BMS permet maintenant de mettre rapidement en ligne des informations sur le secteur de la santé et d'optimiser les opportunités liées à la rapidité du média Internet.

Enfin, nous souhaitons attirer votre attention sur le forum de discussion en ligne, qui s'est enrichi de certaines améliorations fonctionnelles et qui propose aux utilisatrices et aux utilisateurs de discuter et de commenter «en temps réel» les articles publiés dans le BMS, ou d'échanger en général sur des thèmes relatifs à la santé. De mon point de vue, il serait souhaitable que les débats encore (trop) souvent menés dans le courrier des lecteurs de la version papier et étalés sur des semaines, soient transférés vers cette rubrique en ligne, beaucoup plus appropriée.

La base sur laquelle le BMS pourra s'appuyer pour relever les défis d'un avenir médiatique manifestement électronique est donc en place. Je souhaite à toutes les lectrices et à tous les lecteurs de continuer à passer d'agréables moments à nous lire, que ce soit en ligne ou sous forme de version papier «classique».

Bruno Kesseli 